\title{
Evaluación de la reactividad puzolánica de residuos de pizarras del depósito Tchihingue en Angola: Influencia de la composición mineralógica
}

\author{
Assessment of the pozzolanic reactivity of \\ slates waste from the Tchihingue deposit in \\ Angola: Influence of the mineralogical composition
}

\author{
Claudio Fernándes da Mata ${ }^{1}$, Roger Samuel Almenares Reyes ${ }^{2}$, José Antonio Otaño Noguel ${ }^{3}$
}

\footnotetext{
${ }^{1}$ Oficina Provincial para el Desarrollo Económico Integrado. Departamento de Recursos Minerales, Av. de la casa Boni Barrio de Tchavola, 120, Lubango, Huíla, Angola.

${ }^{2}$ Universidad de Moa (UMoa), Departamento de Metalurgia - Química (MG), Av. C. García, 15, CEP: 83330, Moa, Holguín, Cuba.

${ }^{3}$ Universidad de Moa (UMoa), Departamento de Minería (MI), Av. C. García, 15, CEP: 83330, Moa, Holguín, Cuba. e-mail: cfdamata1980@gmail.com, ralmenares@ismm.edu.cu,joseot@ismm.edu.cu
}

\begin{abstract}
RESUMEN
En el presente trabajo se evaluaron residuos de rocas pizarras del depósito Tchihingue en Angola como posible fuente de materiales cementicios suplementarios, enfocado en la reactividad puzolánica y la composición mineralógica de los residuos de pizarra amarilla y pizarra parda. La caracterización química y mineralógica de residuos de pizarras sin calcinar y calcinados, se realizó por Espectrometría de Absorción Atómica (EAA) y difracción de rayos X (DRX), respectivamente. Ambos residuos de pizarras están compuestas por cuarzo y moscovita, y en la pizarra amarilla se identifica caolinita y en la pizarra parda hematita como fases secundarias. La reactividad puzolánica mediante solubilidad de sílice y alúmina en álcali y el índice de actividad de resistencia con cemento Portland, mostró que la pizarra amarilla calcinada presenta la mayor reactividad, lo cual está relacionado con la presencia de caolinita en la muestra, por ser la fase más activa entre los minerales arcillosos. Los residuos de pizarras evaluados cumplen con los requerimientos químicos y físicos-mecánicos, de acuerdo con la norma ASTM C 618, por lo que se considera que ambos residuos calcinados presentan perspectivas para ser empleados como materiales cementicios suplementarios.
\end{abstract}

Palabras-claves: reactividad puzolánica, materiales cementicios, residuos de pizarra, pizarras, puzolana

\begin{abstract}
In the present work, slate rock wastes from the Tchihingue deposit in Angola were assessed as a potential source of supplementary cementitious materials, focused on pozzolanic reactivity and the mineralogical composition of the wastes of yellow and brown slate. Atomic Absorption Spectrometry (EAA) and X-ray diffraction (XRD), respectively were used performed the chemical and mineralogical characterization of uncalcined and calcined slate wastes. Quartz and muscovite are the main phases in both slate wastes. Kaolinite in the yellow slate and hematite in the brown slate were identified as secondary phases. Pozzolanic reactivity through solubility of silica and alumina in alkali and the strength activity index with Portland cement, showed that the calcined yellow slate shows the highest reactivity, which is related to the presence of kaolinite in the sample, as it is the phase most active among clay minerals. The slate wastes evaluated comply with the chemical and physical-mechanical requirements, in accordance with the ASTM C 618 standard, for which reason both calcined wastes are considered to present prospects for use as supplementary cementitious materials.
\end{abstract}

Keywords: pozzolanic reactivity, cementitious materials, slate waste, slate, pozzolan 


\section{INTRODUCCIÓN}

La industria cementera ha estado siguiendo una serie de estrategias para mejorar su sostenibilidad y disminuir las emisiones de $\mathrm{CO}_{2}$ a la atmosfera, dentro de las principales se encuentran el uso de combustibles alternativos, el mejoramiento de la eficiencia energética en la producción de clínquer, la introducción de materias primas alternativas ricas en calcio que no necesitan ser descarbonatadas para fabricación de clínquer, y el reemplazo de una parte del cemento o clínquer con materiales cementicios suplementarios (MCS) [1]. Este último se considera el más efectivo para la reducción de los gases de efecto invernadero (GEI) emitidos por tonelada de cemento y a la vez puede incrementarse la producción de cemento y con menores costos de inversión.

La industria cementera tiene como reto alcanzar la sostenibilidad en el sector. Esta situación ha obligado a buscar nuevos materiales cementicios suplementarios para su comercialización de acuerdo a las normas regulatorias [2]. Una de las vías es el uso de subproductos industriales y desechos de la producción minera que presenten características apropiadas para ser empleadas como adiciones activas al cemento [3]. El amplio rango de residuos mineros, con posibilidades de ser activados para convertirlos de un material inerte a un material cementante suplementario activo [4-7].

La industria de rocas ornamentales genera grandes cantidades de residuos que causan un impacto negativo al medio ambiente y a ecosistemas [8]. Los residuos de esta industria han sido evaluados para su uso como adición mineral [9] y áridos ligeros [10] en hormigones, en la industria cerámica [8, 11, 12] y como material suplementario $[6,13,14]$. El aprovechamiento de los residuos de las rocas ornamentales constituye también una de las líneas de investigación importantes para alcanzar la sostenibilidad de esa industria.

Según una declaración ambiental realizada por la Asociación Galega de Pizarristas (AGP), la pizarra es uno de los productos más sostenibles para cubiertas, si se compara con otros materiales usados para esta aplicación, el fibrocemento y la teja cerámica que emiten más dióxido de carbono y consumen más agua y energía en su producción [15]. Sin embargo, la extracción de pizarra para su uso como material de construcción genera grandes cantidades de residuos (entre $75 \%$ y $90 \%$ en masa), que son vertidos en escombreras, y por consiguiente trae problemas económicos, ambientales y sociales [2, 16-18]. Se han realizado varias investigaciones para el empleo de estos residuos, los cuales se enmarcan fundamentalmente en su valorización como áridos ligeros [19-26], empleo en mejoramiento de pavimento [18, 21, 27, 28] y como materia prima para la industria cerámica $[16,28,29]$. El uso de estos residuos como material puzolánico ha sido poco extendido debido a su baja reactividad [13,30-32]. No obstante, algunos investigadores han demostrado la viabilidad de utilizar residuos de pizarra luego de su activación como materiales puzolánicos en la fabricación de cementos eco-eficientes [2, 33-35], lo cual responde fundamentalmente a la mineralogía de estos residuos y el procesamiento realizado.

En Angola, se prevé la explotación del depósito de pizarra Tchihingue, localizado en la provincia de Huíla. Este depósito presenta dos tipos litológicos de pizarra, una pizarra parda y otra amarilla. El esquema de aprovechamiento integral de las referidas pizarras tiene en cuenta su valorización como material cementicio suplementario para su mezcla con cemento. Sin dudas, esto puede evitar, la acumulación de cantidades significativas de desechos de pizarras que contaminan el ecosistema y hacer de este un producto aún más sostenible. Por lo que, el objetivo de esta investigación es evaluar los residuos de rocas pizarras del depósito Tchihingue como posible fuente de materiales cementicios suplementarios enfocado en la reactividad puzolánica de los dos tipos residuos y la influencia de la composición mineralógica sobre esta.

\section{MATERIALES Y MÉTODOS}

\subsection{Selección de las muestras}

El estudio se enmarca en el área del depósito de pizarras sericítica Tchihingue localizado en el municipio de Humpata en la provincia de Huíla en Angola. El depósito se distingue por dos tipos litológicos fundamentales, una pizarra de color pardo oscura y otra de color amarilla. Durante el proceso de explotación previsto, el residuo queda depositado de forma independiente de acuerdo a su tipo litológico: residuo de pizarra amarilla (RPA) y residuo de pizarra parda (RPP). De cada tipo de residuo se tomó una muestra compósito de $120 \mathrm{~kg}$, las cuales fueron reducidas mediante cuarteo hasta obtener $5 \mathrm{~kg}$ de cada tipo.

\subsection{Preparación de las muestras}

Las muestras $5 \mathrm{~kg}$ correspondiente a cada pizarra, de manera independiente, fue sometida a un proceso de reducción de tamaño mediante dos etapas de trituración en máquinas de mandíbulas, la segunda etapa en ci- 
clo cerrado con una operación de cribado para obtener un tamaño máximo de material a la descarga de 3,15 $\mathrm{mm}$. Se tomaron $400 \mathrm{~g}$ de cada muestra y fueron molidas por separado en un molino de preparación de muestras hasta conseguir una granulometría menor de $0,075 \mathrm{~mm}$ para los ensayos de caracterización mediante composición química y mineralógica. La cantidad de muestra restante fue sometida a un proceso de secado durante $24 \mathrm{~h}$ en una estufa a una temperatura de $110^{\circ} \mathrm{C}$ y posteriormente a un proceso de calcinación a 850 ${ }^{\circ} \mathrm{C}$ durante una hora. Los residuos calcinados fueron identificados de la manera siguiente: residuo de pizarra amarilla calcinado (RPAC) y residuo de pizarra parda calcinado (RPPC). La temperatura de calcinación fue seleccionada de acuerdo a las valoraciones reportadas en la literatura [33, 36, 37].

Una muestra de $500 \mathrm{~g}$ de cada producto calcinado fue molida durante 15 minutos en un molino de bolas de laboratorio para lograr similar distribución de tamaño de partículas (Figura 1) con una superficie específica de $3711,28 \mathrm{~cm}^{2} / \mathrm{g}$ para el RPPC y para el RPAC de $3583,89 \mathrm{~cm}^{2} / \mathrm{g}$, lo cual fue determinada en un analizador de tamaño de partículas HORIBA LA - 910 en condiciones estándar.

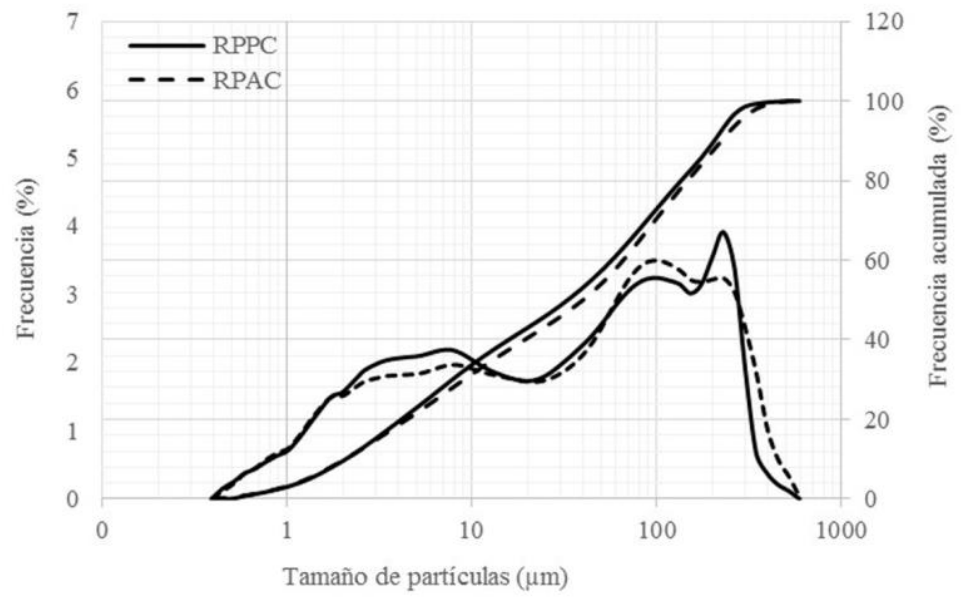

Figura 1: Característica de tamaño del residuo de roca pizarra amarilla y pizarra parda calcinados y molidos.

\subsection{Caracterización de los residuos de pizarras sin calcinar y calcinados}

Los residuos de pizarra y los productos calcinados fueron caracterizados mediante composición química determinada por Espectrometría de Absorción Atómica en un espectrómetro modelo Solar 929 tipo UNYCAM con llama de $\mathrm{N}_{2} \mathrm{O}$-Acetileno para la determinación del aluminio y el silicio, Aire - Acetileno para los demás elementos. La composición mineralógica fue ejecutada mediante Difracción de rayos X (DRX) en un difractómetro $\mathrm{X}^{\prime} \mathrm{PERT}^{3}$ de PANalytical con barrido tipo Gonio en [ $\left.{ }^{\circ} 2 \theta\right]$, registro angular desde 4.0042 hasta 79.9962 , una distancia de paso en ${ }^{\circ} 2 \theta$ de 0.0080 , radiación de $\mathrm{Cu}$ y filtro de níquel y diferencia de potencial es de $40 \mathrm{kV}$ y corriente de $30 \mathrm{~mA}$.

\subsection{Determinación de la reactividad puzolánica de los productos calcinados}

La reactividad de los productos calcinados fue evaluada mediante solubilidad de sílice y alúmina en álcali, teniendo en cuenta el método descrito por He et al. [38] para minerales arcillosos. También se evaluó mediante resistencia a la compresión en morteros normalizados en sistemas cemento - puzolana, de acuerdo con las especificaciones de la norma EN 196-1 [39], con un 30 \% de sustitución de cemento Portland por residuos de pizarra calcinados y se comparó con las especificaciones de la norma ASTM C-618 [40]. El cemento de referencia utilizado fue un cemento Portland de tipo P-35 con $95 \%$ pasado por el tamiz $90 \mu \mathrm{m}$ (Figura 2) y una superficie específica de $2749,25 \mathrm{~cm}^{2} / \mathrm{g}$. 


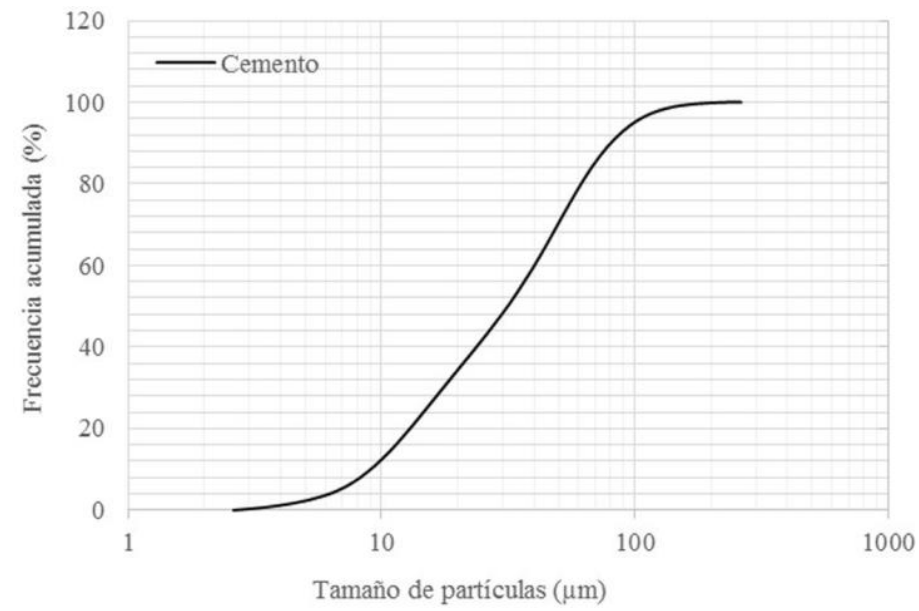

Figura 2: Curva granulométrica del cemento empleado en la elaboración de los morteros.

\section{RESULTADOS Y DISCUSIÓN}

\subsection{Resultados de la caracterización química y mineralógica de los residuos de pizarras}

En las Tabla 1 y 2 se presentan la composición química y mineralógica determinadas mediante difracción de rayos X y el contenido de las principales fases identificadas para el residuo de pizarra amarilla (RPA) y el residuo de pizarra parda (RPP). Los residuos de pizarra son ricos en óxido de silicio y presentan relativamente altos contenidos de aluminio, potasio e hierro, seguidos de cantidades moderadas de sodio, calcio y magnesio. En correspondencia con dicha composición, en la mineralogía de ambos residuos de pizarras se evidencia como fases principales el cuarzo y la moscovita. El residuo de pizarra amarilla se distingue por la presencia de caolinita y el residuo de pizarra parda por la presencia de hematita como fases secundarias.

Se puede notar una deferencia en los contenidos de silicio, aluminio y hierro, lo cual está en correspondencia con la cuantificación de las fases minerales (Tabla 2). El RPA con mayor contenido de $\mathrm{SiO}_{2}$ y menor contenido de $\mathrm{Al}_{2} \mathrm{O}_{3}$ presenta mayor contenido de cuarzo y menor contenido de fases contendedoras de aluminio (46\%) (moscovita $41 \%$ y caolinita $5 \%$ ), mientras que el RPP más rico en $\mathrm{Al}_{2} \mathrm{O}_{3}$ y $\mathrm{Fe}_{2} \mathrm{O}_{3}$ y de menor contenido de $\mathrm{SiO}_{2}$ presenta menor contenido de cuarzo y mayor contenido de fases que contienen aluminio (moscovita $54 \%$ ) y moderado contenido de fase portadora de hierro (hematita).

Tabla 1: Composición química de los residuos de roca pizarra (\%).

\begin{tabular}{c|c|c|c|c|c|c|c|c|c|c}
\hline & $\mathbf{A l}_{2} \mathbf{O}_{3}$ & $\mathbf{S i O}_{2}$ & $\mathbf{F e}_{2} \mathbf{O}_{3}$ & $\mathbf{N a}_{2} \mathbf{O}$ & $\mathbf{K}_{2} \mathbf{O}$ & $\mathbf{C a O}$ & $\mathbf{M g O}$ & $\mathbf{S O}_{3}$ & Otros & PPI \\
\hline RPA & 13,62 & 71,11 & 2,14 & 1,16 & 4,17 & 1,12 & 1,35 & 0,28 & 0,22 & 4,82 \\
\hline RPP & 15,66 & 66,48 & 4,63 & 1,40 & 5,02 & 0,98 & 1,31 & 0,29 & 0,69 & 3,54 \\
\hline
\end{tabular}

Tabla 2: Principales fases identificadas mediante difracción de rayos X.

\begin{tabular}{l|c|c}
\hline & FASES PRINCIPALES & CONTENIDO DE LAS FASES (\%) \\
\hline \multirow{4}{*}{ RPA } & Cuarzo & 54 \\
\cline { 2 - 3 } & Moscovita & 41 \\
\cline { 2 - 3 } & Caolinita & 5 \\
\hline \multirow{4}{*}{ RPP } & Cuarzo & 43 \\
\cline { 2 - 3 } & Moscovita & 54 \\
\cline { 2 - 3 } & Hematita & 3 \\
\hline
\end{tabular}

\subsection{Resultados de la caracterización química y mineralógica de los residuos de pizarras calcinados}

La composición química de los residuos de pizarra amarilla calcinada (RPAC) y de pizarra parda calcinada (RPPC) se presenta en la Tabla 3. Esta composición es consistente con la composición química de los residuos sin calcinar. Los resultados muestran que ambas pizarras cumplen con los requerimientos químicos 
$\left(\mathrm{SiO}_{2}, \mathrm{Al}_{2} \mathrm{O}_{3}, \mathrm{Fe}_{2} \mathrm{O}_{3}>70 \%\right)$ y el \% $\mathrm{SO}_{3}$ inferior al $4 \%$, de acuerdo con la norma ASTM C 618 [40] para las puzolanas clase $\mathrm{N}$ puzolanas naturales calcinadas y sin calcinar. Independientemente de que se ha reportado que los álcalis presentes en algunas pizarras evaluadas como puzolana, no presentan un efecto directo sobre la actividad puzolánica [7, 41], es necesario tener en cuenta el relativamente elevado contenido de álcalis en los residuos de pizarras aquí analizados, en particular, si se pretende emplear en hormigones que contienen áridos reactivos o el cemento con el que va a ser mezclado encuentra limitaciones en el contenido de álcalis.

Tabla 3: Composición química de los residuos de roca pizarra calcinados (\%).

\begin{tabular}{l|c|c|c|c|c|c|c|c|c|c}
\hline & $\mathbf{A l}_{2} \mathbf{O}_{\mathbf{3}}$ & $\mathbf{S i O}_{\mathbf{2}}$ & $\mathbf{F e}_{2} \mathbf{O}_{\mathbf{3}}$ & $\mathbf{N a}_{\mathbf{2}} \mathbf{O}$ & $\mathbf{K}_{\mathbf{2}} \mathbf{O}$ & $\mathbf{C a O}$ & $\mathbf{M g O}$ & $\mathbf{S O}_{3}$ & Otros & PPI \\
\hline RPAC & 15,97 & 71,36 & 2,30 & 1,23 & 4,56 & 1,29 & 1,47 & 0,12 & 0,58 & 1,13 \\
\hline RPPC & 17,02 & 67,26 & 5,00 & 1,45 & 5,11 & 1,13 & 1,36 & 0,13 & 0,36 & 1,16 \\
\hline
\end{tabular}

La composición mineralógica de los residuos de pizarras calcinados a $850{ }^{\circ} \mathrm{C}$ (RPAC y RPPC) en comparación con el patrón de difracción de los residuos de pizarra sin tratamiento térmico (RPA y RPP), se presentan en las Figuras 3 y 4, respectivamente. Ambos materiales calcinados parecen mantener su similitud con la composición inicial, aunque los máximos de los filosilicatos fundamentalmente, disminuyen la intensidad, lo que muestra el efecto de la temperatura de calcinación, a la que fue sometido, sobre su estructura. Otro indicador cualitativo, del efecto del tratamiento térmico, está en el ligero incremento del halo amorfo de los difractogramas, poco más acentuado para el RPAC. Es importante destacar que en el RPAC desaparecen los máximos asignados a la caolinita (Figura 3), lo cual es un comportamiento que está en correspondencia con las trasformaciones que exhibe este mineral a la temperatura de calcinación sometida y se encuentra en los reportes de la literatura [42-44]. La moscovita es afectada parcialmente durante la calcinación a la referida temperatura, evidenciándose trazas de leucita para la muestra de RPAC y trazas de sanidina en el RPPC, lo que está en correspondencia con los reportes de que la moscovita se descompone entre los 800 y $900{ }^{\circ} \mathrm{C}$, aproximadamente [45-47].
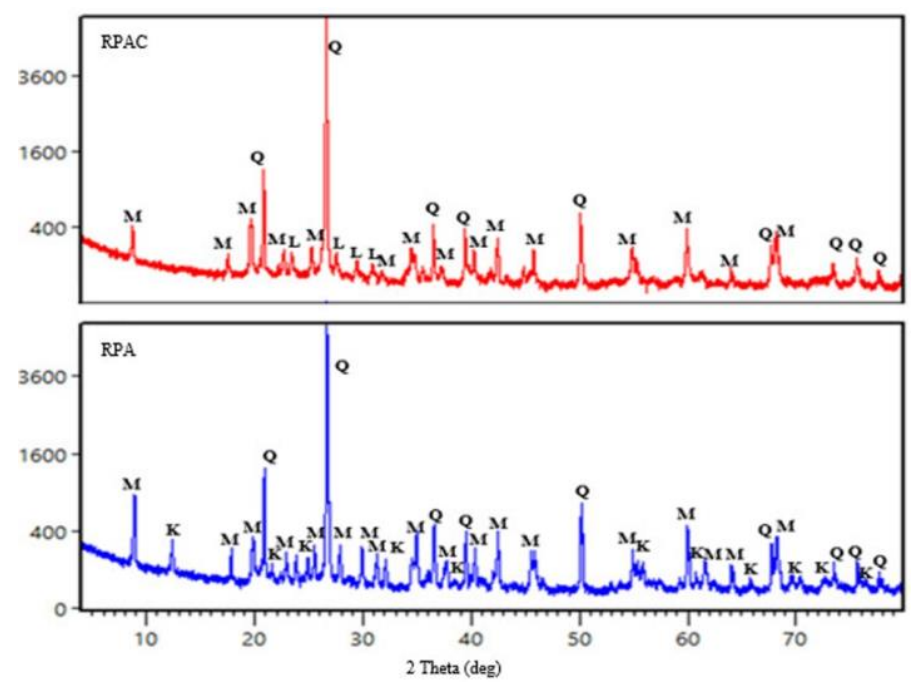

Figura 3: Composición mineralógica del residuo de pizarra amarilla calcinada y la muestra natural. 

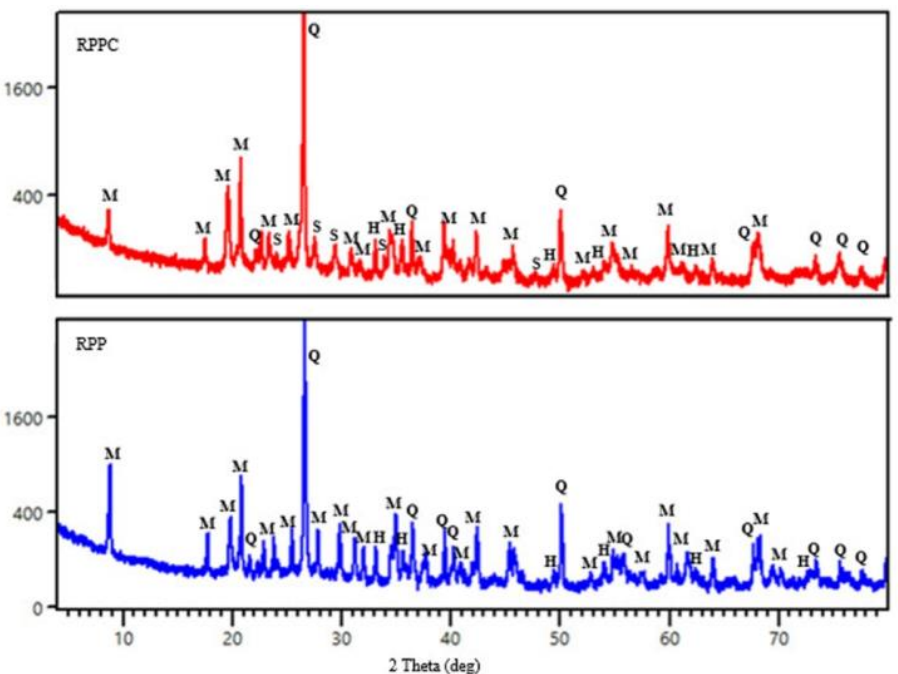

Figura 4: Composición mineralógica del residuo de pizarra parda calcinada y de la muestra natural.

\subsection{Reactividad según la solubilidad alcalina}

En la Figura 5, se muestra la solubilidad de la sílice y alúmina en álcali. Las pizarras calcinadas a $850{ }^{\circ} \mathrm{C}$ muestran que el silicio tiende a disolverse más que el aluminio, lo cual está relacionado con las fases activas en estas muestras. De forma general, la solubilidad de ambos componentes es mayor para el RPAC que para el RPPC, lo cual favorece la reactividad de la primera. Este comportamiento es coherente con la composición mineralógica de los residuos de pizarras. El RPA que contiene caolinita, mineral que al ser activado térmicamente es el de mayor reactividad entre todos los minerales arcillosos, aporta mayor cantidad de componentes activos, incluso es donde mayor contenido de alúmina disuelto se observa, a pesar de que es el de menor contenido de alúmina en la muestra en comparación con el RPPC que no contiene caolinita, pues la hoja octaédrica es la que mayor afectación recibe durante el proceso de activación térmica, ocurriendo una completa deshidroxilación a la temperatura de calcinación estudiada [43, 44, 48].

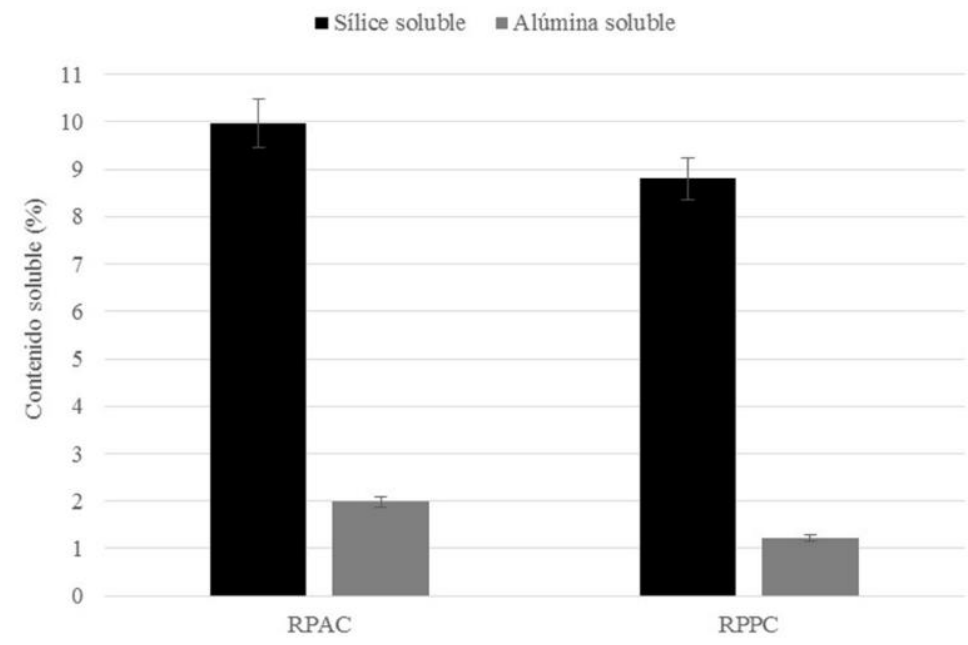

Figura 5: Sílice y alúmina solubles en álcali.

\subsection{Comportamiento físico-mecánico de los sistemas mezclados}

En la Figura 6 se muestran los valores de resistencia a la compresión en morteros normalizados con un $30 \%$ de sustitución de cemento P-35 por los productos de calcinación a $850{ }^{\circ} \mathrm{C}$ de los residuos de pizarras calcinadas. Como valores de referencia se utilizan los resultados de resistencia a la compresión de una serie con $100 \%$ de P-35.

A los tres días se observa un predominio del efecto físico sobre la resistencia mecánica. En este caso, la resistencia a la compresión se incrementa en la medida en que lo hace la superficie específica $(3711,28$ 
$\mathrm{cm}^{2} / \mathrm{g}$ el RPPC y $3583,89 \mathrm{~cm}^{2} / \mathrm{g}$ el RPAC). Las partículas finas, en particular, aquellas con mayor superficie específica actúan como centro de nucleación para los productos de hidratación y favorece el crecimiento a edades tempranas de los productos de hidratación del cemento Portland [49]. Por tanto, aquí las diferencias entre las dos series con incorporación de residuos de pizarras calcinadas parecen ser una consecuencia de la diferencia en la superficie específica.

A partir de los siete días, los valores de resistencia a la compresión de los morteros con incorporación de pizarra amarilla calcinada sobrepasan los valores de resistencia de los morteros con productos calcinados de la pizarra parda, lo cual está relacionado con la diferencia en el material reactivo, que a estas edades ejerce mayor efecto. De forma general, este comportamiento se corresponde con los resultados de solubilidad alcalina (RPAC $>$ RPPC), mostrando que la reactividad puzolánica comienza a ser significativa a partir de los siete días y su diferencia está mayormente determinada por la presencia de caolinita en la muestra de pizarra amarilla, mineral arcilloso de mayor reactividad [43, 48, 50].

La resistencia a la compresión de todas las series con adición de los productos de calcinación de residuos de pizarra se mantiene por debajo de los valores obtenidos con la serie de referencia. Sin embargo, los índices de actividad de resistencia calculados (Tabla 4), cumplen con los criterios de actividad puzolánica expresados en la norma ASTM C 618 [40], por tanto se considera a ambos residuos calcinados con perspectivas de ser empleados como materiales cementicios suplementarios.

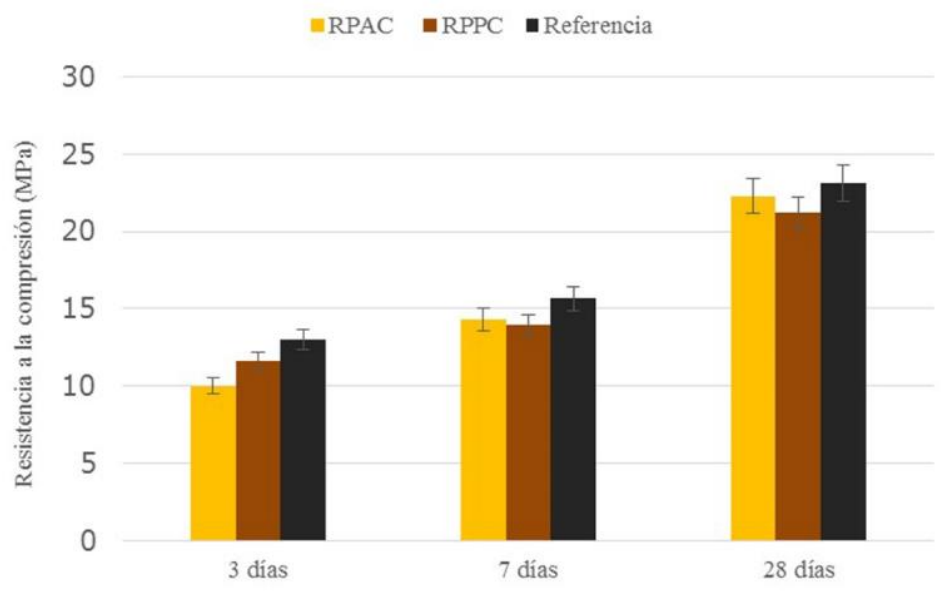

Figura 6: Evolución de la resistencia a la compresión de los morteros con incorporación de pizarras calcinadas.

Tabla 4: Índice de actividad de resistencia.

\begin{tabular}{c|c|c}
\hline \multirow{2}{*}{} & ÍNDICE DE ACTIVIDAD DE RESISTENCIA (\%) \\
\cline { 2 - 3 } & RPAC & RPPC \\
\hline 3 días & 76,92 & 89,23 \\
\hline 7 días & 91,45 & 88,92 \\
\hline 28 días & 96,33 & 91,58 \\
\hline
\end{tabular}

\section{CONCLUSIONES}

Se evaluaron los residuos de rocas pizarras del depósito Tchihingue en Angola para su utilización en la producción de materiales cementicios suplementarios, de lo cual se concluye:

Los residuos de pizarras están compuestos esencialmente por cuarzo y moscovita. En la pizarra amarilla se identifica caolinita y en la pizarra parda la hematita, como fases secundarias.

La composición química de los residuos de pizarras evaluados cumplen con los requerimientos quími$\cos \left(\mathrm{SiO}_{2}, \mathrm{Al}_{2} \mathrm{O}_{3}, \mathrm{Fe}_{2} \mathrm{O}_{3}>70 \%\right)$ y el \% $\mathrm{SO}_{3}$ inferior al $4 \%$, de acuerdo con la norma ASTM C 618 para las puzolanas clase $\mathrm{N}$ puzolanas naturales calcinadas y sin calcinar. Si el contenido de álcali se convierte en un requisito obligatorio, es necesario hacer análisis adicionales para conocer su influencia.

El residuo de pizarra amarilla calcinada presenta la mayor solubilidad de silicio y aluminio en álcali, 
lo cual está directamente relacionado con la presencia de caolinita en la muestra; fase más afectada durante el proceso de calcinación a $850{ }^{\circ} \mathrm{C}$ y que aporta mayor cantidad de componentes activos entre los minerales arcillosos.

A los tres días, el comportamiento físico - mecánico de los morteros que contienen residuo de pizarra calcinada obedece a la superficie específica. A partir de los siete días, el comportamiento está relacionado con la composición mineralógica de las pizarras, con índice de actividad de resistencia superior para el residuo de pizarra calcinada amarilla que contiene caolinita.

\section{AGRADECIMENTOS}

Los autores del trabajo agradecen al equipo del Laboratorio del Centro de Investigaciones del Níquel (CEDINIQ), por su profesionalidad y atención en la ejecución de los ensayos químicos y mineralógicos.

\section{BIBLIOGRAFIA}

[1] IEA/CSI-WBCSD, “Technology Roadmap: Low-Carbon Transition in the Cement Industry 2060,” París, 2018 .

[2] FRÍAS, M., VIGIL, R., GARCÍA, R., et al., "Scientific and technical aspects of blended cement matrices containing activated slate wastes," Cement \& Concrete Composites, v. 48, pp. 19-25, Apr. 2014.

[3] SÁNCHEZ DE ROJAS, M.I., FRÍAS, M., "Natural and artificial pozzolans in eco-efficient concrete," in Eco-efficient concrete, F. Pacheco, S. Jalali, J. Labrincha, and V. John, Eds. Cambridge (UK): Woodhead Publishing Series in Civil and Structural Engineering, pp. 83-104, 2013.

[4] VIGIL DE LA VILLA, R., FRÍAS, M., SÁNCHEZ DE ROJAS, M.I., et al., "Mineralogical and morphological changes of calcined paper sludge at different temperatures and retention in furnace," Applied Clay Science, v. 36, n. 4, pp. 279-286, May. 2007.

[5] FRÍAS, M., GARCÍA, R., VIGIL, R., et al., "Calcination of art paper sludge waste for the use as a supplementary cementing material," Applied Clay Science, v. 42, n. 1-2, pp. 189-193, Dec. 2008.

[6] MEDINA, G., SÁEZ DEL BOSQUE, I.F., FRÍAS, M., et al., "Mineralogical study of granite waste in a pozzolan $/ \mathrm{Ca}(\mathrm{OH}) 2$ system: influence of the activation process," Applied Clay Science, v. 135, pp. 362-371, Jan. 2017.

[7] IRASSAR, E.F., BONAVETTI, V.L., Castellano, C. C., et al., "Calcined illite-chlorite shale as supplementary cementing material: Thermal treatment, grinding, color and pozzolanic activity," Applied Clay Science, v. 179, p. 105143, Jan. 2019.

[8] ALMEIDA, K.S., SOARES, R.A.L., MATOS, J.M.E., "Efeito de resíduos de gesso e de granito em produtos da indústria de cerâmica vermelha: revisão bibliográfica," Revista Matéria, v. 25, n. 01, p. e-12568, Apr. 2020.

[9] DIETRICH, Y.P., TELES, C.R., VIEIRA, G.L., "Desempenho mecânico e análise da corrosão das armaduras em concretos produzidos com adição de resíduos de rochas ornamentais," Revista Matéria, v. 22, n. 04, p. e-11891, Oct. 2017.

[10] SOUZA, N.S.L., ANJOS, M.A.S., SÁ, M.V.V.A., et al., "Desenvolvimento de agregados leves a partir de resíduo de corte de pedras ornamentais ( granitos e mármores ) e argila," Revista Matéria, v. 25, n. 01, p. e-12559, Apr. 2020.

[11] CHINELATTO, A.S.A., CHINELATTO, A.L., FERREIRA, D.E.M., et al., "Estudo da viabilidade do uso de resíduo de polimento de rochas ornamentais em porcelanas," Revista Matéria, v. 20, n. 04, pp. 10751086, Dec. 2015.

[12] JUNIOR, P.F.S., NETO, B.B.P., OLIVEIRA, M.A.B., "Cerâmica vermelha incorporada com resíduo de pedras ornamentais tratada termicamente," Brazilian Journal of Development, v. 6, n. 9, pp. 72545-72561, Sep. 2020.

[13] SILVA, B., SOUZA, L., BAPTISTELLA, J., et al., "Influence of the heterogeneity of waste from wet processing of ornamental stones on the performance of Portland cement composites," Construction and Building Materials, v. 262, p. 120036, Nov. 2020.

[14] MEDINA, G., SÁEZ DEL BOSQUE, I.F., FRÍAS, M., et al., "Granite quarry waste as a future ecoefficient supplementary cementitious material (SCM): scientific and technical considerations," Journal of Cleaner Production, v. 148, pp. 467-476, Apr. 2017. 
[15] COBAS, M., "Un estudio constata que la pizarra es el producto más sostenible para cubiertas,” La Voz de Galicia, Galicia, 2013.

[16] PALHARES, L.B., GOUVÊA, C., BINDA, F.F., et al., "Characterization of Slate Powder Wastes from Minas Gerais - Brazil,” Key Engineering Materials, v. 848, pp. 10-19, Jun. 2020.

[17] SILVA, R.K.R., "Propostas de aproveitamento de resíduos de ardósia da cidade de pompéu, Minas Gerais," Revista Intercâmbio, v. 6, pp. 86-95, 2015.

[18] ANTUNES, M.A.G., GUIMARÃES, A.C.R., MARQUES, G.L.O., "Uma solução viável para a reciclagem de resíduos industriais de ardósia em pavimentação," Revista Ibero-Americana de Ciências Ambientais, v. 11, n. 4, pp. 236-254, Jun, Jul. 2020.

[19] SADEK, M.M., ISMAIL, M.K., HASSAN, A.A.A., "Stability of Lightweight Self-Consolidating Concrete Containing Coarse and Fine Expanded Slate Aggregates," ACI Materials Journal, v. 117, n. 3, pp. 133-143, May. 2020.

[20] ROSA, M.P., BORGES, M.A., NASCIMENTO, L.G., et al., "Viabilidade na reutilização da ardósia em substituição do agregado graúdo na dosagem do concreto," Brazilian Journal of Development, v. 6, n. 1, pp. 936-948, Sep. 2020.

[21] SANTOS, S.C.P., SOARES DE LIMA, J.L., FIGUEIREDO, L.H.A., et al., "Análise da aplicação de rejeitos de ardósia em solo natural para pavimentação," Revista Intercâmbio, v. 11, pp. 230-240, 2018.

[22] KHAN, M.A., AHMED, I., BASIT, A., et al., "Use of slate in making lightweight concrete," IOP Conf. Series: Materials Science and Engineering, v. 414, n.1, pp. 012007, Apr. 2018.

[23] SURANENI, P., FU, T., AZAD, V.J., et al., "Pozzolanicity of finely ground lightweight aggregates," Cement and Concrete Composites, v. 88, pp. 115-120, Apr. 2018.

[24] OMAR, A.T., ISMAIL, M.K., AND HASSAN, A.A.A., "Use of Polymeric Fibers in the Development of Semilightweight Self-Consolidating Concrete Containing Expanded Slate," Journal of Materials in Civil Engineering, v. 32, n. 5, p. 04020067 , May. 2020.

[25] KHARTABIL, A., MARTINI, S.A.L., "Thermal Transmission Properties of Sustainable Concrete with Supplementary Cementitious Materials,” Key Engineering Materials, v. 853, pp. 142-149, Jul. 2020.

[26] MO, K.H., LING, T., ALENGARAM, U.J., et al., "Overview of supplementary cementitious materials usage in lightweight aggregate concrete," Construction and Building Materials, v. 139, pp. 403-418, May. 2017.

[27] LÚCIO, R., ANTÔNIO, V., RODRIGUES, C.D.S., et al., "Avaliação do efeito da adição de resíduo de ardósia no módulo de resiliência de misturas com solo argiloso,” Transportes, v. 27, n. 4, pp. 145-158, 2019.

[28] OTI, J.E., KINUTHIA, J.M., SNELSON, D.G., et al., "Applications of slate waste material in the UK," Waste and Resource Management, v. 163, n. 1, pp. 9-15, Feb. 2010.

[29] MANSUR, A.A.P., PERES, A.E.C., PALHARES, L., et al., "Study of pore size distribution of slate ceramic pieces produced by slip casting of waste powders," Minerals Engineering, v. 19, n. 5, pp. 525-527, Apr. 2006.

[30] LIMA, S.C.L., COSTA, L.C.B., DEFÁVERI, K.C.S., et al., "Study on Assessment of Pozzolanic Activity: Slate Cutting Waste," ACI Materials Journal, v. 117, n. 3, pp. 3-10, May. 2020.

[31] SCHUAB, M.R., COSTA, V.M., SERPA, A.C.L., et al., "Study of mechanical and durability properties of mortars using slate waste," International Journal of Development Research, v. 10, no. 05, pp. 3608936095, May. 2020.

[32] COTTA, L.S., “Avaliação da atividade pozolânica do resíduo de corte de ardósia,” M.Sc., Universidade Federal de Ouro Preto - Escola de Minas, Ouro Petro, Brasil, 2017.

[33] FRÍAS, M., VIGIL DE LA VILLA, R., GARCÍA, R., et al., "The Influence of Slate Waste Activation Conditions on Mineralogical Changes and Pozzolanic Behavior," Journal of American Ceramic Society, v. 96, n. 7, pp. 2276-2282, May. 2013.

[34] SILVA, M.E.M.C., PERES, A.E.C., "Thermal expansion of slate wastes," Minerals Engineering, v. 19, n. 5, pp. 518-520, Apr. 2006.

[35] MIRANDA, F.F., "Estudo da influência do tratamento termo-mecânico nas propriedades do resíduo de ardósia," Tesis de M.Sc., Universidade Federal de Minas Gerais, Belo Horizonte, Brasil, 2018.

[36] SÁNCHEZ-SOTO, P.J., RUIZ-CONDE, A., BONO, R., et al., “Thermal evolution of a slate,” Journal 
of Thermal Analysis and Calorimetry, v. 90, n. 1, pp. 133-141, Jul. 2007.

[37] SÁEZ DEL BOSQUE, I.F., FRÍAS, M., ROJAS, M.I.S., et al., "Activation temperature-mediated mineralogical transformations in slate quarry sludge: Pozzolanic properties," Construction and Building Materials, v. 187, pp. 819-829, Oct. 2018.

[38] HE, C., MAKOVICKY, E., OSBACK, B., "Thermal stability and pozzolanic activity of calcined kaolin,” Applied Clay Science, v. 9, n. 3, pp. 165-187, Sep. 1994.

[39] EN 196-1, “Methods of Testing Cement - Part 1: Determination of Strength”. European Committee for Standardization, 2016.

[40] ASTM C 618, "Standard specification for coal fly ahs and raw or calcined pozzolan for use as a mineral admixture in concrete,"ASTM, 2015.

[41] MITTRI, S.H.M., DEGEN, M.K., VIEIRA, G.L., et al., "Assessment of the pozzolanic activity of ornamental stone waste after heat treatment and its effect on the mechanical properties of concretes," Revista Ibracon de Estructuras e Materiais, v. 11, n. 6, pp. 1186-1207, Dec. 2018.

[42] ILIĆ, B., RADONJANIN, V., MALEŠEV, M., et al., "Effects of mechanical and thermal activation on pozzolanic activity of kaolin containing mica,” Applied Clay Science, v. 123, pp. 173-181, Apr. 2016.

[43] HOLLANDERS, S., ADRIAENS, R., SKIBSTED, J., et al., "Pozzolanic reactivity of pure calcined clays," Applied Clay Science, v. 132-133, pp. 552-560, Nov. 2016.

[44] ALUJAS, A., FERNÁNDEZ, R., QUINTANA, R., et al., "Pozzolanic reactivity of low grade kaolinitic clays : Influence of calcination temperature and impact of calcination products on OPC hydration," Applied Clay Science, v. 108, pp. 94-101, May. 2015.

[45] Földvári, M., Handbook of thermogravimetric system of minerals and its use in geological practice: Occasional Papers of the Geological Institute of Hungary, v. 213. Budapest, Hungary: Geological Institute of Hungary, 2011.

[46] GRIDI-BENNADJI, F., BENEU, B., LAVAL, J.P., et al., "Structural transformations of Muscovite at high temperature by X-ray and neutron diffraction," Applied Clay Science, v. 38, n. 3-4, pp. 259-267, Feb. 2008.

[47] LECOMTE, G. L., BONNET, J.P., AND BLANCHART, P., "A study of the influence of muscovite on the thermal transformations of kaolinite from room temperature up to $1,100{ }^{\circ} \mathrm{C}$," Journal of Materials Science, v. 42, n. 20, pp. 8745-8752, Jul. 2007.

[48] DANNER, T., NORDEN, G., AND JUSTNES, H., "Characterisation of calcined raw clays suitable as supplementary cementitious materials,” Applied Clay Science, v. 162, pp. 391-402, Sep. 2018.

[49] BERODIER, E., SCRIVENER, K., "Understanding the Filler Effect on the Nucleation and Growth of CS-H,” Journal of the American Ceramic Society, v. 97, n. 12, pp. 3764-3773, Aug. 2014.

[50] FERNÁNDEZ, R., MARTIRENA, F., SCRIVENER, K.L., "The origin of the pozzolanic activity of calcined clay minerals: A comparison between kaolinite, illite and montmorillonite," Cement and Concrete Research, v. 41, n. 1, pp. 113-122, Jan. 2011.

\section{ORCID}

Claudio Fernándes da Mata

Roger Samuel Almenares Reyes

José Antonio Otaño Noguel http://orcid.org/0000-0002-8422-0830

http://orcid.org/0000-0002-7185-7330

http://orcid.org/0000-0002-3586-113X 